Supporting Information for "Thin Films of Polyethylenimines and Alkyltrimethylammonium Bromides at the Air/Water Interface” by O'Driscoll, B.M.D.; Milsom, E.; Fernandez-Martin, C.; White, L.; Roser, S.J. and Edler, K.J.

\title{
Brewster Angle Microscopy
}
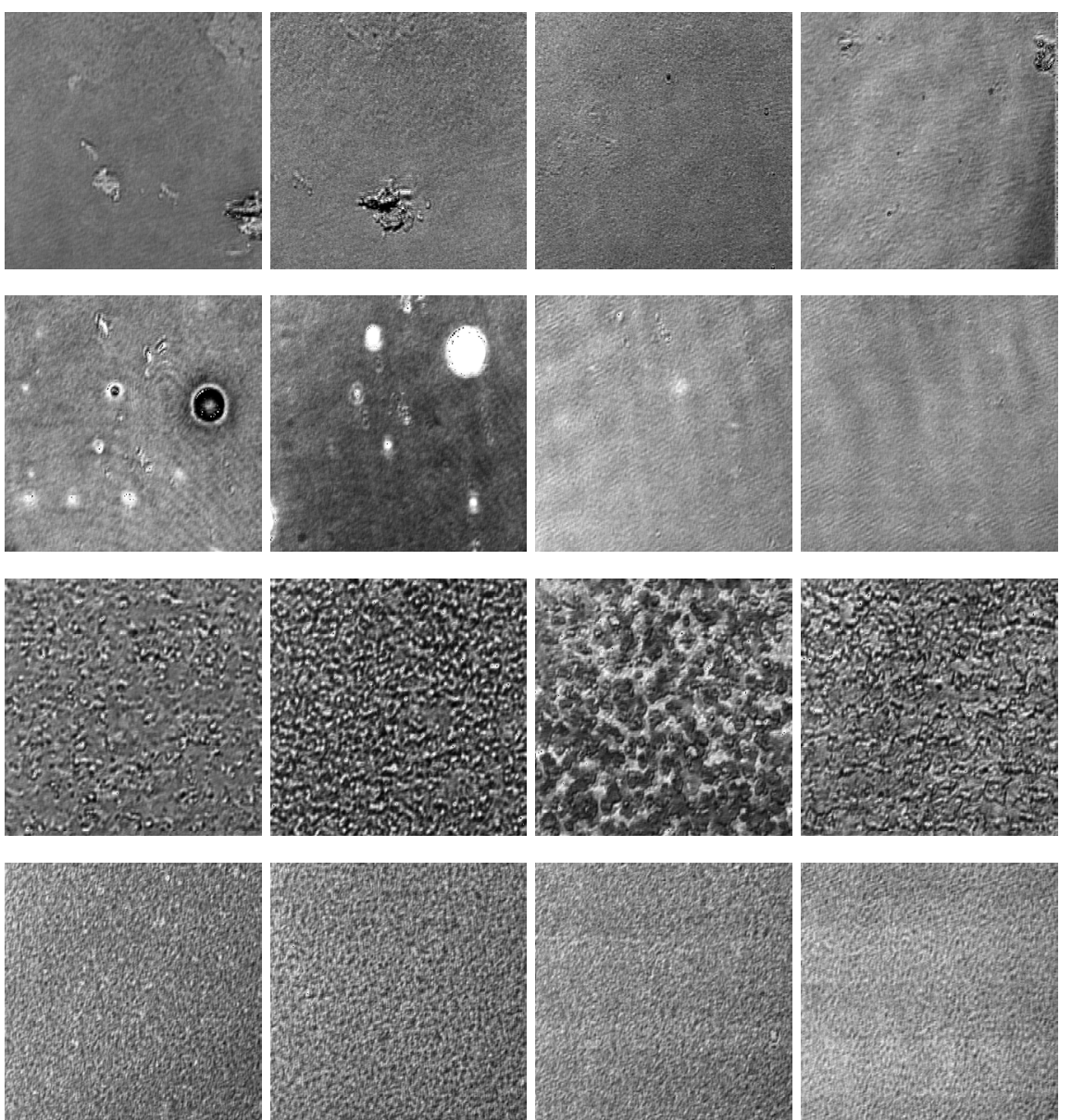

Figure 1. BAM images of $\mathrm{C}_{\mathrm{n}} \mathrm{TAB} / \mathrm{PEI}$ films taken (left to right) at 5, 10, 20 and 30 minutes after mixing for (top to bottom) $\mathrm{C}_{16} \mathrm{TAB}$ long $\mathrm{PEI}$ conc. $1 / 4, \mathrm{C}_{12} \mathrm{TAB}$ long PEI conc. 1, $\mathrm{C}_{14} \mathrm{TAB}$ short PEI conc. 1/16, and $\mathrm{C}_{12} \mathrm{TAB}$ short PEI conc. 1/2. Each image represents an area of $225 \times 225 \mu \mathrm{m}$.
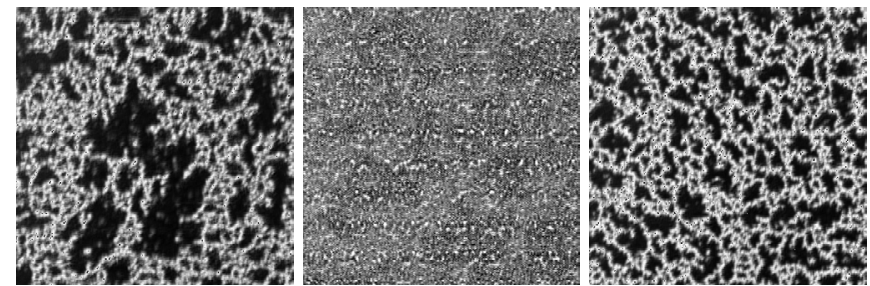

Figure 2. BAM images of a $\mathrm{C}_{16} \mathrm{TAB}$ short PEI Ratio $1 / 2$ solution 5 minutes after mixing (left), 30 minutes after mixing (middle) and 5 minutes after the solution had been covered for $2 \frac{1}{2}$ hours (right). 
pH Titration

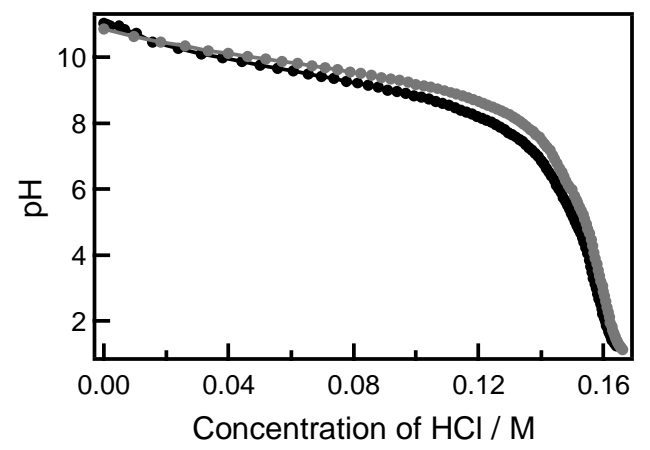

Figure 3. $\mathrm{pH}$ titrations of concentrated hydrochloric acid with long PEI (black) and short PEI (grey).

\section{Small Angle Neutron Scattering}
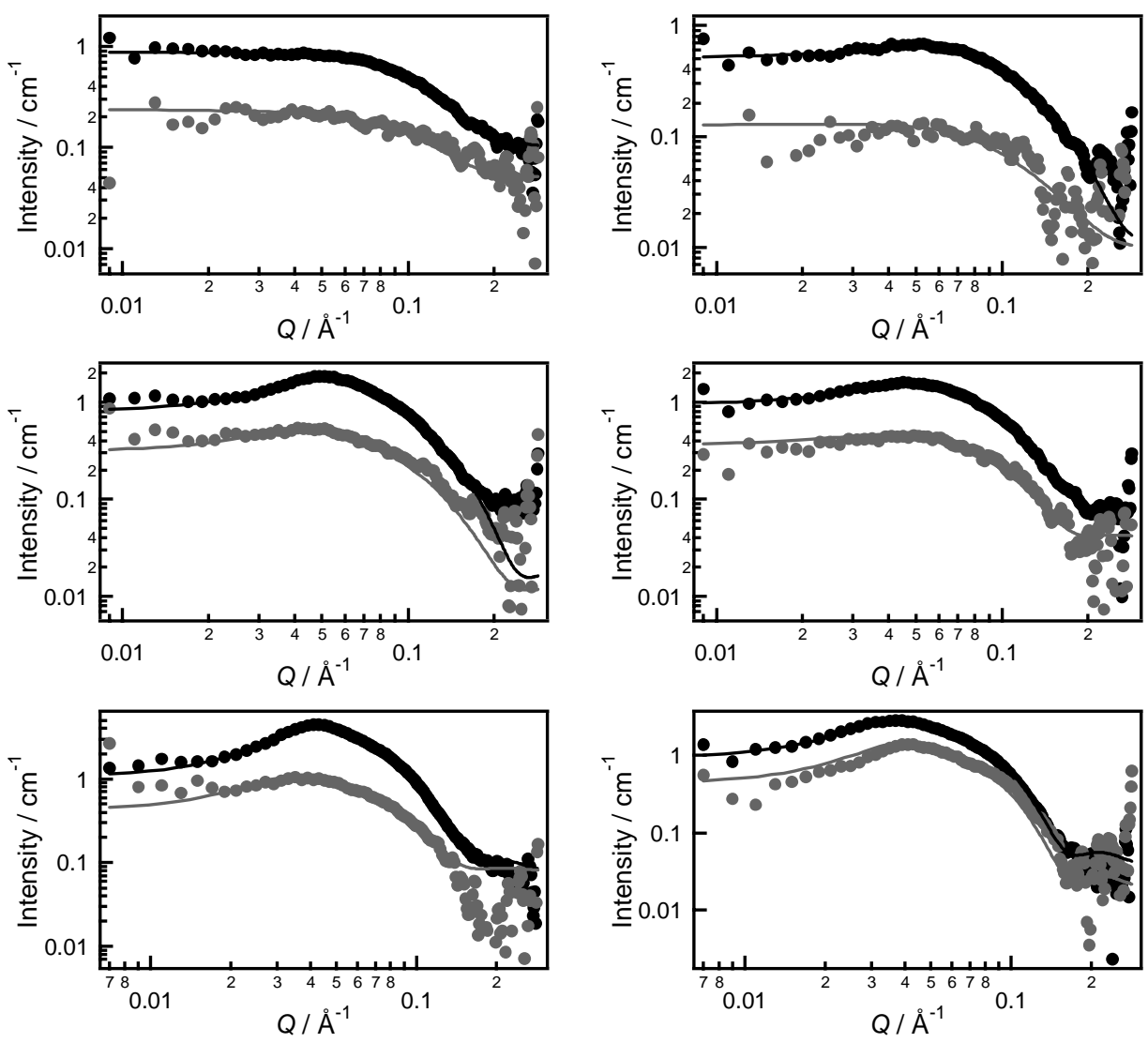

Figure 4. Sample of the SANS curves (markers) and fits (lines) for the $\mathrm{C}_{\mathrm{n}} \mathrm{TAB} / \mathrm{PEI}$ systems. Left to right $-\mathrm{C}_{12} \mathrm{TAB}$ long PEI conc. $1, \mathrm{C}_{12} \mathrm{TAB}$ short PEI conc. 1, $\mathrm{C}_{14} \mathrm{TAB}$ long PEI conc. $3 / 4, \mathrm{C}_{14} \mathrm{TAB}$ short PEI conc. 1/2, $\mathrm{C}_{16} \mathrm{TAB}$ long PEI conc. $1 / 2$, and $\mathrm{C}_{16} \mathrm{TAB}$ short PEI conc. $1 / 8$. The protonated surfactant/ $100 \% \mathrm{D}_{2} \mathrm{O}$ runs are shown in black and the deuterated surfactant/ $40 \% \mathrm{D}_{2} \mathrm{O}$ runs are shown in grey. 


\begin{tabular}{|c|c|c|c|}
\hline $\mathrm{C}_{12}$ TAB short PEI & $\begin{array}{l}\text { Width of Micelle } \\
(\AA)\end{array}$ & 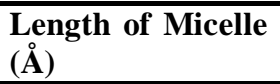 & Micelle Shape \\
\hline $\begin{array}{l}\text { Ratio } 1 \\
\text { Ratio } 3 / 4 \\
\text { Ratio } 1 / 2 \\
\text { Ratio } 1 / 4 \\
\text { Ratio } 1 / 8\end{array}$ & $\begin{array}{l}27.9 \\
20.8 \\
31.4 \\
29.6 \\
25.0\end{array}$ & $\begin{array}{l}64.2 \\
72.5 \\
52.5 \\
52.8 \\
58.3\end{array}$ & $\begin{array}{l}\text { Elliptical } \\
\text { Elliptical } \\
\text { Elliptical } \\
\text { Elliptical } \\
\text { Elliptical }\end{array}$ \\
\hline \multicolumn{4}{|l|}{$\mathrm{C}_{12} \mathrm{TAB}$ long PEI } \\
\hline $\begin{array}{l}\text { Ratio } 1 \\
\text { Ratio } 3 / 4 \\
\text { Ratio } 1 / 2 \\
\text { Ratio } 1 / 4 \\
\text { Ratio } 1 / 8\end{array}$ & $\begin{array}{l}48.5 \\
49.3 \\
50.5 \\
52.0 \\
45.3\end{array}$ & $\begin{array}{l}24.6 \\
29.4 \\
29.9 \\
27.1 \\
44.7\end{array}$ & $\begin{array}{l}\text { Disk } \\
\text { Disk } \\
\text { Disk } \\
\text { Disk } \\
\text { Spherical }\end{array}$ \\
\hline \multicolumn{4}{|l|}{$\mathrm{C}_{14} \mathrm{TAB}$ short PEI } \\
\hline $\begin{array}{l}\text { Ratio } 1 \\
\text { Ratio } 3 / 4 \\
\text { Ratio } 1 / 2 \\
\text { Ratio } 1 / 4 \\
\text { Ratio } 1 / 8 \\
\end{array}$ & $\begin{array}{l}32.5 \\
41.1 \\
47.6 \\
53.9 \\
48.3\end{array}$ & $\begin{array}{l}71.1 \\
58.6 \\
47.6 \\
29.3 \\
48.1\end{array}$ & $\begin{array}{l}\text { Elliptical } \\
\text { Elliptical } \\
\text { Spherical } \\
\text { Disk } \\
\text { Spherical }\end{array}$ \\
\hline \multicolumn{4}{|l|}{$\mathrm{C}_{14} \mathrm{TAB}$ long PEI } \\
\hline $\begin{array}{l}\text { Ratio } 1 \\
\text { Ratio } 3 / 4 \\
\text { Ratio } 1 / 2 \\
\text { Ratio } 1 / 4 \\
\text { Ratio } 1 / 8\end{array}$ & $\begin{array}{l}30.7 \\
33.0 \\
34.9 \\
38.4 \\
39.7\end{array}$ & $\begin{array}{l}77.3 \\
75.7 \\
74.5 \\
67.7 \\
64.8\end{array}$ & $\begin{array}{l}\text { Elliptical } \\
\text { Elliptical } \\
\text { Elliptical } \\
\text { Elliptical } \\
\text { Elliptical }\end{array}$ \\
\hline \multicolumn{4}{|l|}{$\mathrm{C}_{16} \mathrm{TAB}$ short PEI } \\
\hline $\begin{array}{l}\text { Ratio } 1 \\
\text { Ratio } 3 / 4 \\
\text { Ratio } 1 / 2 \\
\text { Ratio } 1 / 4 \\
\text { Ratio } 1 / 8 \\
\end{array}$ & $\begin{array}{l}41.9 \\
48.7 \\
46.8 \\
47.6 \\
48.2\end{array}$ & $\begin{array}{l}82.1 \\
76.5 \\
76.5 \\
76.7 \\
72.9\end{array}$ & $\begin{array}{l}\text { Elliptical } \\
\text { Elliptical } \\
\text { Elliptical } \\
\text { Elliptical } \\
\text { Elliptical }\end{array}$ \\
\hline \multicolumn{4}{|l|}{$\mathrm{C}_{16}$ TAB long PEI } \\
\hline $\begin{array}{l}\text { Ratio } 1 \\
\text { Ratio } 1 / 2 \\
\text { Ratio } 1 / 4 \\
\text { Ratio } 1 / 8\end{array}$ & $\begin{array}{l}42.9 \\
48.5 \\
50.7 \\
51.6\end{array}$ & $\begin{array}{l}77.0 \\
73.0 \\
69.8 \\
68.8\end{array}$ & $\begin{array}{l}\text { Elliptical } \\
\text { Elliptical } \\
\text { Elliptical } \\
\text { Elliptical }\end{array}$ \\
\hline
\end{tabular}

Table 1. Total micellar widths and lengths (in Angstroms), and shape of the micelles formed in solutions of $\mathrm{C}_{\mathrm{n}} \mathrm{TAB}$ with PEI as determined by fitting of SANS data. 


\section{Neutron Reflectometry}
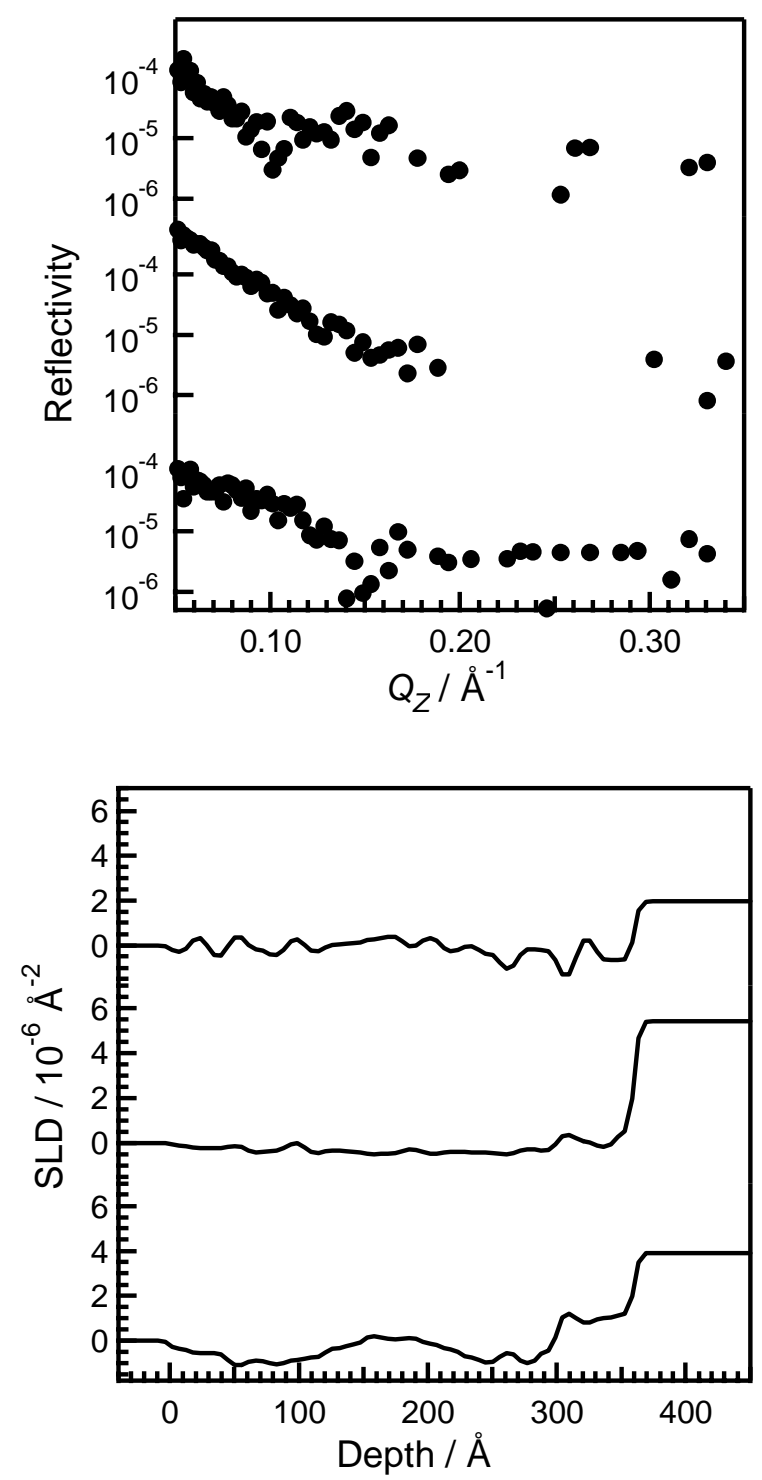

Figure 5. Neutron reflectometry profiles of (top to bottom) $\mathrm{C}_{12} \mathrm{TAB}$ short PEI conc. 1, $\mathrm{C}_{12} \mathrm{TAB}$ short PEI conc. $1 / 16$, and $\mathrm{C}_{14} \mathrm{TAB}$ short PEI conc. 1/16.
Figure 6. Scattering Length Density (SLD) profiles of (top to bottom) $\mathrm{C}_{12} \mathrm{TAB}$ short $\mathrm{PEI}$ conc. 1, $\mathrm{C}_{12} \mathrm{TAB}$ short PEI conc. $1 / 16$, and $\mathrm{C}_{14} \mathrm{TAB}$ short PEI conc. 1/16. $\mathrm{D}_{2} \mathrm{O}$ has a SLD of $6.366 \times 10^{-6} \AA^{-2}$. 